\title{
Reflexiones sobre la trayectoria y evolución en Proyectos de Módulo. Reflections on the path and evolution in module projects.
}

\author{
I. López-Forniés ${ }^{1}$, J.M.Agudo ${ }^{2}$, A.Biedermann ${ }^{1}$, B.Sánchez-Valverde ${ }^{3}$, A.Pardina ${ }^{4}$ \\ ignlopez@unizar.es,jmagudo@unizar.es, anna@unizar.es, belensv@unizar.es, apardina@unizar.es
}

\author{
${ }^{1}$ Diseño y Fabricación \\ Universidad de Zaragoza \\ Zaragoza, España
}

\author{
${ }^{2}$ Dirección y Organización de Empresas \\ Universidad de Zaragoza \\ Zaragoza, España
}

\author{
${ }^{3}$ Métodos Estadísticos \\ Universidad de Zaragoza \\ Zaragoza, España
}

\author{
${ }^{4}$ Ingeniería Eléctrica \\ Universidad de Zaragoza \\ Zaragoza, España
}

\begin{abstract}
Resumen- Durante los últimos 8 años en la titulación de Grado en Ingeniería de Diseño Industrial y Desarrollo de Producto de la Universidad de Zaragoza se han realizado una serie de experiencias docentes llamadas Proyecto de Módulo en las que se consigue una integración de materias y conocimientos transversales aplicados a proyectos de diseño y desarrollo de producto. En este trabajo se presenta una reflexión sobre el origen de este tipo de proyecto y la trayectoria de estas experiencias discutiendo acciones emprendidas y resultados obtenidos en esta experiencia docente, destacando aspectos como la integración y aplicación de conocimientos, la organización de grupos docentes, la gestión de grupos de trabajo de alumnos y los sistemas de evaluación, entre otros.
\end{abstract}

Palabras clave: Innovación docente, Proyecto de módulo, Competencias en diseño, Conocimiento transversal.

Abstract- During the past 8 years in the degree in Engineering of Industrial Design and Product Development of the University of Zaragoza a series of educational experiences have been realized, called "module project" in which an integration of subjects and transversal knowledge applied to projects for Design and Product Development. This paper presents a reflection on the origin of this type of project and the trajectory of these experiences discussing actions undertaken and results obtaines of this type of teaching experience, highlighting aspects such as the integration and application of knowledge, organization of teaching groups, Management of student work groups, evaluation systems, among others.

Keywords: Teaching innovation, Module project, Competences in design, Transversal knowledge.

\section{INTRODUCCIÓN}

El origen de la implantación de proyectos de modulo se produce con el cambio de titulación de Ingeniería Técnica en Diseño Industrial al Grado en Ingeniería en Diseño Industrial y Desarrollo de Producto en la Universidad de Zaragoza en el curso 2009-2010. Son dos hechos particulares los que propician la implantación, el primero la autoevaluación de la anterior titulación en el que se detecta "Falta de coherencia entre Plan de estudios, programa formativo y perfiles de egreso" como una debilidad que implica que los perfiles del egresado y sus conocimientos no se ajustan a las necesidades de las empresas.

El segundo que en una encuesta (Lopez-Fornies \& Manchado Pérez, 2008) realizada a egresados de titulaciones de Diseño de diversas universidades españolas se detecta que los conocimientos científico-técnicos y la formación profesionalizante están bastante mal valoradas en cuanto a su aplicación e idoneidad en sus actividades laborales. Por estas razones se comenzó a trabajar en proyectos de diseño que integrasen conocimientos transversales y que el alumno trabajase de una manera interdisciplinar aplicando conocimientos de otras áreas de conocimiento a su proyecto de diseño.

Por otra parte, la Universidad de Zaragoza en ese momento había comenzado a desarrollar una serie de acciones para mejorar el sistema de aseguramiento de la garantía de calidad de las titulaciones, aplicando cambios en la adquisición y evaluación de las competencias alineados con la transición al EEES, Espacio Europeo de Educación Superior.

El desarrollo de estos proyectos se inició con una motivación clave, la mejora del aprendizaje de conocimientos y competencias transversales en diseño, trabajando en aprendizaje basado en proyectos (PBL) y manteniendo un carácter integrador de las materias no específicas de diseño.

Los Proyectos de Módulo (PM) son trabajos en los que varias asignaturas del título proponen objetivos particulares que sirven para conseguir un objetivo común y se caracterizan por ser trabajos colaborativos e interdisciplinares en los que se desarrollan y evalúan varias competencias básicas. El aprendizaje por módulos se ha desarrollado en varias universidades nacionales e internacionales en distintos ámbitos que pasan por todas las ramas de conocimiento (Padilla Arias, 2012; Zelaieta Anta, 2012; Rekalde Rodríguez, 2012; Manchado Pérez, 2012; Serrano Tierz et al., 2013).

En esta comunicación presentamos la trayectoria de 8 años de trabajo con Proyectos de Módulo, la evolución de los mismos, los resultados de la experiencia y una reflexión sobre los logros, acciones de mejora y futuras líneas de trabajo.

\section{CONTEXTO}

La experiencia con PM se inició en el curso 2009-10, teniendo apoyo en las convocatorias de Proyectos de Innovación Docente de la Universidad de Zaragoza (PIIDUZ_09_2_127, PIIDUZ_10_3_438, PIIDUZ_11_3_482, PIIDUZ_14_073, PIIDUZ_14_069, 2016 PIIDUZ_15_023, PIIDUZ_16_369). Ese mismo año se implantaba el segundo curso del Grado y se realizó un proyecto en cada cuatrimestre. 
Los proyectos de módulo pretenden una serie de mejoras en innovación docente:

- $\quad$ Estructurar el plan de estudios en bloques de asignaturas que son la base para los siguientes bloques.

- Desarrollar un trabajo común y amplio en vez de realizar muchos pequeños trabajos. Se integran los requisitos exigidos y se busca un tema o trabajo que satisfaga varias materias al mismo tiempo.

- Equilibrar de tareas a lo largo del cuatrimestre, evitando picos excesivos de trabajo.

- Comprender el carácter multidisciplinar del diseño y la necesidad de trabajar diferentes competencias en un mismo proyecto.

En el presente trabajo, reflexionamos sobre el PM que se realiza en el segundo cuatrimestre del Grado. Las asignaturas involucradas son: Taller de Diseño III: Creatividad, Diseño Industrial aplicado al Producto, Estadística y Fiabilidad de Producto, Aspectos Económicos y Empresariales del Diseño y Tecnología Eléctrica y Electrónica.

El PM consiste en el desarrollo completo de un producto. Lo desarrollan grupos de 4 estudiantes y corresponde a un $30 \%$ de la calificación de cada asignatura. Los estudiantes presentan públicamente su producto al grupo de profesores y realizan una maqueta y el correspondiente packaging.

Con este tipo de proyectos el alumno percibe unidad y continuidad que se desarrolla a lo largo del cuatrimestre en varias asignaturas, en el que se establecen diversas revisiones y es necesario realizar una correcta secuenciación de contenidos.

Además, se realiza una evaluación conjunta por parte del grupo de profesores, valorando el logro del proyecto y no la suma de las partes, esta acción implica la voluntad y el acuerdo de los docentes a dedicar parte de su evaluación a un trabajo común y, finalmente, el PM aumenta la comunicación entre los profesores permitiendo un flujo de conocimiento y obliga a establecer una coordinación y supervisión del proyecto.

\section{DESCRIPCIÓN}

En la Tabla 1 se puede observar la trayectoria del grupo de docentes.

A lo largo de 8 años académicos se han realizado 7 proyectos de innovación docente y se ha trabajado de manera específica por parte de los alumnos la adquisición de competencias transversales como el trabajo en equipo, la gestión de grupos, la coordinación de proyectos y las presentaciones visuales y orales.

Por parte del equipo docente se han trabajado aspectos como la coordinación, seguimiento y comunicación de grupos de trabajo, gestión de proyectos, medida y mejora de la calidad, la integración de conocimientos, la facilitación de la adquisición de competencias transversales, la mejora de la calidad de la docencia, la mejora de la evaluación y su objetividad, o el reparto equilibrado de trabajos y la gestión de potenciales problemas.

De entre los resultados más destacables podemos decir que la cohesión del grupo de profesores es el factor más importante para dar continuidad a un proyecto de 8 años. También se contó en varias ocasiones con expertos en innovación docente del ICE y de ANECA, que ayudaron en la definición de estrategias y acciones particulares para conseguir los objetivos de los proyectos de módulo.

También hay que resaltar la participación de especialistas del mundo de la empresa y el diseño que ven con ojos limpios los trabajos de los alumnos y que destacan la buena resolución del proyecto para su complejidad por su imaginación y presentación visual, pero detectan carencias en aspectos relacionados con la materialización del mismo en cuanto a la realidad de la empresa, quizá debido al momento de sus estudios en el que se encuentran, aun no muy maduros y con carencias en aspectos de desarrollo y fabricación.

El PM nos ha permitido evolucionar en dos direcciones, la primera al aumentar año a año el nivel de los trabajos exigidos a los alumnos, tanto a nivel del trabajo global como a nivel individual materia a materia, y la segunda en la propia gestión de los proyectos por parte de alumnos y profesores.

En el primer caso, la experiencia se implantó el primer curso con un proyecto cuyo diseño implicaba poca dificultad para tratar de asegurar el funcionamiento del grupo anteponiéndolo al resultado de diseño. El ejercicio inicial fue el diseño de una linterna. En años posteriores se incrementó la dificultad con el diseño de pequeños electrodomésticos y en los últimos años se plantean dispositivos en los que desarrollar una función como la inducción al sueño o el entrenamiento mental.

Estos últimos productos son más abstractos y permiten un mayor nivel creativo y de innovación a la vez que elevan la dificultad en el resto de materias como la orientación al usuario y el mercado, su presentación y comercialización o el diseño de su parte técnica. En este sentido, sirve de ejemplo, entre otras incorporaciones en las distintas materias, el hecho de que en los últimos años los alumnos han incorporado encuestas de investigación empresarial entre usuarios potenciales y entrevistas en profundidad con expertos, para identificar necesidades no satisfechas y potenciales públicos objetivos, para posteriormente desarrollar un prototipo funcional del circuito eléctrico, hacer una simulación o un montaje en Arduino.

Se ha observado que al aumentar el nivel de exigencia ha aumentado el nivel de la calidad de los trabajos de los alumnos y la competitividad entre grupos de alumnos, este hecho también puede estar condicionado por la presentación pública de los trabajos o la exposición de posters y maquetas.

Respecto a la mejora en la gestión de los proyectos cabe destacar que los alumnos han ido mejorando y son más capaces en la gestión del tiempo, la organización del trabajo, el reparto de tareas y la consciencia respecto a la evaluación gracias a la implantación de las actas y las rubricas.

Respecto a la mejora de la gestión del proyecto de módulo por parte de los profesores ha evolucionado al trabajar sobre un mismo brief que se ha ido ajustando y puliendo a lo largo de los años afinando los objetivos globales y particulares.

Además, el uso de rubricas se ha desarrollado año a año haciéndolas más completas y fáciles de comprender por parte del alumno. 
Como resultado de estos proyectos se han realizado comunicaciones a congresos para divulgación de las diferentes experiencias desarrolladas (Agudo, 2011; Biedermann, 2015; López-Forniés, 2015 y Agudo (2017)).

\section{RESUltados}

Los PM están sirviendo para comenzar una actividad integradora e interdisciplinar entre docentes y que es beneficiosa para el alumno, dado que el aprendizaje ha mejorado.

En esta situación nos planteamos si el esfuerzo realizado es valioso, y solo podemos responder por medio de las encuestas de satisfacción de los alumnos, por los comentarios de los expertos externos en calidad de la enseñanza y por los comentarios de los expertos externos en el ámbito de la empresa.

Los datos recogidos en las encuestas contestadas por los alumnos a lo largo de los años han evidenciado satisfacción y el nivel se ha mantenido a lo largo de los años. Se muestran a continuación en las Figuras 1 y 2 los resultados de las preguntas consideradas más representativas de la satisfacción de los estudiantes (se muestra la información de los 7 últimos cursos en los que la encuesta ha sido la misma, el primer curso fue una encuesta diferente):

1. La coordinación con los componentes de mi grupo ha sido satisfactoria

2. La coordinación de los profesores del módulo ha sido satisfactoria

3. La coordinación de los profesores con respecto a las entregas parciales del trabajo de módulo me parece adecuada.

4. El trabajo de módulo me ha servido para integrar y relacionar los conocimientos de las 5 asignaturas

5. Es mejor realizar un trabajo de módulo a que cada asignatura solicite realizar su propio trabajo independiente del resto.

6. Grado de satisfacción general con trabajo de módulo.

La respuesta es individual, anónima y con posibilidad de puntuar entre 1 y 7.

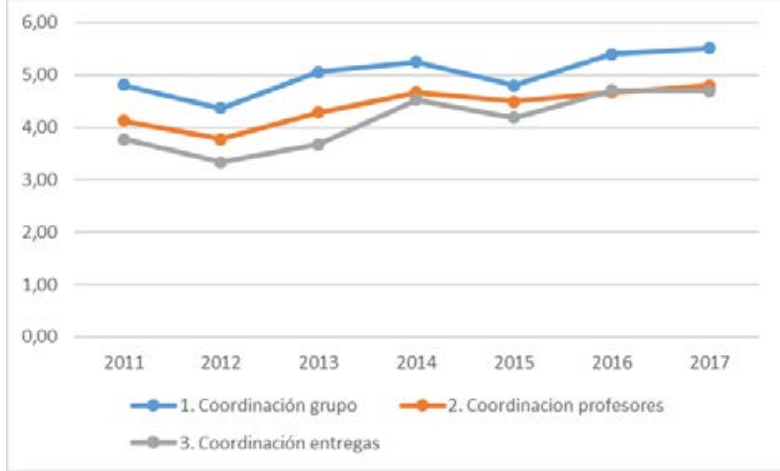

Figura 1: Evolución de la media de las respuestas a las preguntas 1,2 y 3

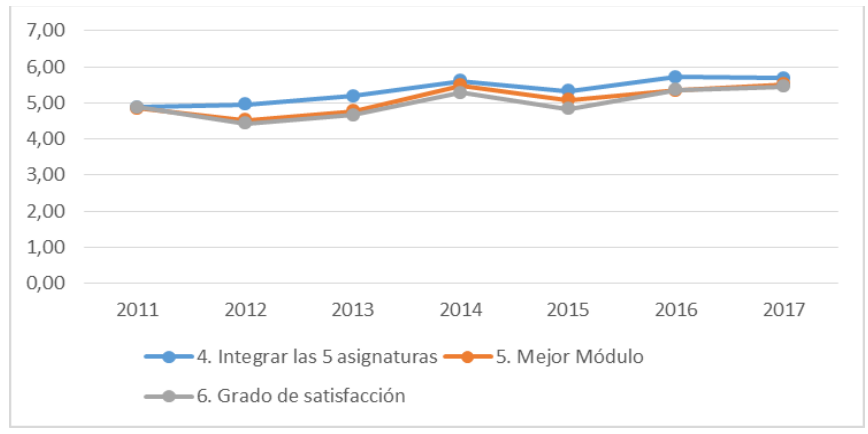

Figura 2: Evolución de la media de las respuestas a las preguntas 4, 5 y 6

La Tabla 2 muestra el incremento medio que se observa entre el primer y último curso (el actual). En todas las cuestiones se observa un aumento de la puntuación media obtenida en los diferentes conceptos.

Tabla 2. Aumento medio en las valoraciones.

\begin{tabular}{|l|c|}
\hline \multicolumn{1}{|c|}{ Pregunta } & $\begin{array}{c}\text { Aumento } \\
\mathbf{2 0 1 1 - 2 0 1 7}\end{array}$ \\
\hline 1. Coordinación grupo & 0,15 \\
\hline 2. Coordinacion profesores & 0,16 \\
\hline 3. Coordinación entregas & 0,24 \\
\hline 4. Integrar las 5 asignaturas & 0,16 \\
\hline 5. Mejor Módulo & 0,14 \\
\hline 6. Grado de satisfacción & 0,12 \\
\hline
\end{tabular}

En todos los aspectos considerados se observa un aumento favorable, aunque también se aprecia potencial de mejora que puede no haberse obtenido por la propia evolución del proyecto y el aumento de nivel de dificultad en los proyectos realizados por los alumnos.

Los comentarios de los expertos externos (ambos en educación y empresa) son positivos y han ayudado a plantear nuevos objetivos y acciones de mejora año a año. Estos datos y comentarios se deben convertir en indicadores para medir el avance anual, pero plantea un problema de definición ya que cada año el proyecto es distinto y los indicadores se deben definir como independientes del PM.

Es importante plantear la cuestión de cómo percibe el alumno las asignaturas de conocimiento transversal y su utilidad en la aplicación a proyectos de diseño. Si en el origen los conocimientos científico-técnicos y de formación básica eran mal valorados y percibidos como poco útiles, llegándose a presentar en el propio trabajo de módulo y su defensa ante el tribunal de profesores como partes aisladas, ahora son percibidos por parte de los alumnos como integrados y relacionados con el diseño.

Otra cuestión a debatir es si el proyecto de módulo afecta a la nota del alumno, y en caso de hacerlo si beneficia su resultado académico.Para resolver esta cuestión se han ido recopilando datos de las notas y evaluaciones de los alumnos en los últimos tres años, los datos aún no están procesados pero una observación preliminar parece indicar que la nota del alumno mejora gracias al proyecto de modulo y que por las respuestas de las encuestas el $30 \%$ es una parte representativa dentro de la evaluación para dicha carga de trabajo y que los alumnos la perciben como correcta. 
Por otra parte, tal y como se muestra en la Tabla 1, después de 8 cursos hemos probado diferentes acciones que no han dado resultado y otras acciones que han permanecido, ya que han demostrado ser valiosas para la buena marcha del PM. Las acciones puestas en marcha y que forman parte de nuestro bagaje actual son:

- Formación de grupos homogéneos.

- Redacción clara de un brief en el que se explica con detalle el trabajo a realizar a lo largo del cuatrimestre.

- Gestión de entregas parciales y organización sin solapes de las 5 asignaturas, tanto en el PM como en el resto.

- Presentación intermedia del trabajo realizado y resolución y corrección de posibles problemas en el PM.

- Presentación final a la que asisten expertos externos y se evalúa mediante rúbrica el PM.

- Conocer mediante encuestas la satisfacción de los estudiantes en diferentes aspectos del PM y seguir mejorando las carencias que se detectan

- Gestión mediante actas de los grupos en sus reuniones, tutor asignado a cada grupos y entrevistas con los grupos cuando se detectan problemas.

- Exposición de paneles y maquetas en la que todos los grupos pueden ver el trabajo de los demás.

\section{CONCLUSIONES}

La continuidad del equipo de profesores en el trabajo de módulo, la revisión anual de los resultados y la definición de acciones de mejora para cada año han sido definitivas para los buenos resultados de la experiencia, siempre teniendo en cuenta que se plantea como una actividad de mejora continua y que para poder continuar es necesario definir con mayor precisión los indicadores.

La satisfacción de los alumnos es alta y el trabajo se percibe por su parte con agrado, pero de nuevo es necesario generar indicadores más precisos y no solo basados en encuestas. La situación ideal sería el poder tener un indicador que tuviera en cuenta los resultados académicos de los alumnos y su satisfacción, generando un indicador o métrica de carácter cualitativo y cuantitativo.

Tras una trayectoria de 8 años es un momento en el que observar los resultados y realizar una reflexión para replantear los objetivos, sin descuidar los logros alcanzados, y permita trabajar otros aspectos más concretos como pueden ser las competencias transversales o competencias específicas.

Finalmente, se plantea como un ejercicio a desarrollar en el futuro una posible encuesta a egresados para conocer si su percepción de las materias científico-técnicas y las básicas son positivas y las valoran como conocimiento que todo ingeniero de diseño debe conocer y dominar para aplicarlas en sus proyectos profesionales.

\section{REFERENCIAS}

Agudo, J. M., López-Forniés, I., Pardina, A., SánchezValverde, B. and Sierra, J. (2011). Docencia por Módulos de asignaturas del Grado de Ingeniería de Diseño Industrial y Desarrollo de Producto. Seguimiento y Mejora. XV International Congress on Project Ingenieering. Huesca.

Agudo, J. M., Bierermann, A. López-Forniés, I., Pardina, A., Sánchez-Valverde, B. (2017). Development of tools for internal control and leadership recognition in working groups. XV International Congress on Project Ingenieering. 3rd International Conference on Higher Education Advances. HEAD_17. Valencia.

Biedermann, A. (2015). SFM Implementation in Product Design Projects with derived specifications. ATIONS. In XXV International Conference on Graphics Engineering. San Sebastián.

López-Forniés, I., Agudo, J. M., Sánchez-Valverde, B., Biedermann, A., y Pardina, A. (2015). Aseguramiento de la Calidad en el aprendizaje modular. P. Acuña (Ed.), Vectores de la pedagogía docente actual. ACCI (Asoc. Cultural y Científica Iberoameric.). Madrid.

López-Forniés, I., Manchado, E. (2008). Estudio de necesidades reales del Mercado Laboral aplicados a la correcta elaboración de los Proyectos Docentes en el Grado de Diseño Industrial y Desarrollo de Producto. PIECYT_08_2_415. Zaragoza.

Manchado, E. López-Forniés, I., (2012). Coordinación por módulos de asignaturas en el Grado de Ingeniería de Diseño Industrial y Desarrollo de Producto de la UZ. Revista de Docencia Universitaria, 10(3), 195-207.

Padilla Arias, A (2012). El sistema modular de enseñanza: una alternativa curricular de educación superior universitaria en México. Revista de Docencia Universitaria, 10(3), 71-98.

Rekalde I., Martínez, B. and Marko, J. I. (2012). Los Proyectos Interdisciplinares de Módulo: Una experiencia innovadora en el Grado de Educación Social de la UPV/EHU. Revista de Docencia Universitaria, 10(3), 209-237.

Serrano Tierz, A., Hernández Giménez, M., Pérez Sinusía, E., \& Biel Ibáñez, P. (2013). Trabajo por módulos: un modelo de aprendizaje interdisciplinar y colaborativo en el Grado en Ingeniería en Diseño Industrial y Desarrollo de Producto. Revista de Docencia Universitaria, 11, 197-220.

Zelaieta, E., Camino, I., Aristizábal, P. and Goñi, E. (2012). El inicio de la experiencia interdisciplinar en la E. U. de Magisterio de Vitoria-Gasteiz: El módulo de profesión docente. Revista de Docencia Universitaria, 10(3), 239262. 
Tabla 1. Evolución de los Proyectos de Módulo. Objetivos, acciones y resultados

\begin{tabular}{|c|c|c|c|}
\hline AÑO & OBJETIVO & ACCIÓN & RESULTADO \\
\hline \multirow{3}{*}{2010} & Integrar objetivos asigntauras & $\begin{array}{l}\text { Realización de un único proy ecto que integra tareas de } \\
\text { distintas asignaturas. }\end{array}$ & $\begin{array}{c}\text { Los alumnos desarrollan distintas competencias y } \\
\text { perciben que se integran y relacionan los conocimientos. }\end{array}$ \\
\hline & $\begin{array}{l}\text { Aumentar la comunicación entre profesores y mejorar } \\
\text { coordinación. }\end{array}$ & $\begin{array}{c}\text { Reunión al principio del curso para plantear los objetivos de } \\
\text { proyecto. }\end{array}$ & La coordinación presenta posibilidades de mejora. \\
\hline & Evaluar la satisfacción de alumnos. & Realización de encuestas al inicio y final del cuatrimestre. & $\begin{array}{l}\text { Las mejoras introducidas año a año se basan en las } \\
\text { respuestas de las encuetas. }\end{array}$ \\
\hline \multirow{4}{*}{2011} & Integrar todas la asignaturas desde principio del proyecto. & $\begin{array}{c}\text { Participación de todos los profesores en la presentación } \\
\text { intermedia. }\end{array}$ & $\begin{array}{l}\text { Mayor integración de conocimientos y orientación para } \\
\text { los alumnos sobre cómo seguir con el proy ecto. }\end{array}$ \\
\hline & $\begin{array}{l}\text { Aumentar la comunicación entre profesores y mejorar } \\
\text { coordinación. }\end{array}$ & $\begin{array}{l}\text { Establecer criterios de evaluación, estructura de proy ecto, } \\
\text { secuenciación de la docencia y de las materias. }\end{array}$ & $\begin{array}{l}\text { Introducir una pregunta sobre coordinación de los } \\
\text { profesores. }\end{array}$ \\
\hline & $\begin{array}{l}\text { Equilibrar el porcentaje de nota del trabajo de módulo en } \\
\text { cada asignatura. }\end{array}$ & $\begin{array}{c}\text { Adaptación de contenidos correspondientes a cada asignatura } \\
\text { y limitación del tiempo destinado al proy ecto en cada } \\
\text { asignatura. }\end{array}$ & $\begin{array}{c}\text { Los cambios introducidos acercan la carga percibida por } \\
\text { los alumnos al 30\% de asignatura, el previsto en el } \\
\text { módulo. }\end{array}$ \\
\hline & $\begin{array}{l}\text { Reparto equilibrado de tareas a lo largo del cuatrimestre, } \\
\text { evitando cargas excesivas de trabajo. }\end{array}$ & $\begin{array}{l}\text { Corrección del calendario de entregas, presentaciones y } \\
\text { exámenes, haciendo un rep arto equilibrado }\end{array}$ & $\begin{array}{l}\text { La distribución de carga de trabajo propuesta por el } \\
\text { equipo docente se percibe equilibrada. }\end{array}$ \\
\hline \multirow{5}{*}{2012} & Mejorar la creación de grupos de los estudiantes. & $\begin{array}{l}\text { Creación de grupos antes del inicio del semestre, limitar los } \\
\text { grupos a } 4 \text { personas. }\end{array}$ & $\begin{array}{l}\text { Grupos más homogéneos. Inicio inmediato de la docencia } \\
\text { y del proyecto. }\end{array}$ \\
\hline & $\begin{array}{l}\text { Mejorar el seguimiento de trabajo en grupo, evitar la } \\
\text { "rémora” que se beneficia del trabajo en grupo. }\end{array}$ & $\begin{array}{c}\text { Control en la presentación intermedia e intervención en el caso } \\
\text { de problemas. Valoración al final de curso de la carga de } \\
\text { trabajo realizada los estudiantes y por ellos mismos. }\end{array}$ & $\begin{array}{c}\text { Ajuste de la nota final de los miembros del grupo tanto } \\
\text { por su excelente trabajo, o por la falta de implicación } \\
\text { suficiente. }\end{array}$ \\
\hline & $\begin{array}{l}\text { Repartir las tareas a lo largo del cuatrimestre, evitar cargas } \\
\text { excesivas de trabajo. }\end{array}$ & $\begin{array}{l}\text { Seguimiento de la dedicación al proyecto en horas por parte } \\
\text { del grupo de trabajo, a lo largo de las } 14 \text { semanas dedicadas al } \\
\text { proy ecto. }\end{array}$ & $\begin{array}{l}\text { Gráficas de evolución del proyecto, evidencias de picos } \\
\text { de trabajo. }\end{array}$ \\
\hline & Garantizar may or objetividad de la evaluación. & $\begin{array}{l}\text { Comunicar si el proyecto es apto o no, la nota se publica una } \\
\text { vez vistos todos los proyectos. Se crea una rúbrica. }\end{array}$ & $\begin{array}{c}\text { Revisión de calificaciones y mejor evaluación al tener una } \\
\text { visión global. }\end{array}$ \\
\hline & Mejorar el proceso de evaluación. & $\begin{array}{c}\text { Presentaciones intermedia y final de proyectos de los } \\
\text { alumnos, evaluación final apoyada en profesionales externos a } \\
\text { la Universidad de Zaragoza. }\end{array}$ & $\begin{array}{c}\text { La presentación intermedia mejora el control del proyecto } \\
\text { y la evaluación continua. Los colaboradores externos } \\
\text { aportan una visión global. }\end{array}$ \\
\hline \multirow[t]{2}{*}{2013} & Mejorar el proceso de evaluación. & $\begin{array}{l}\text { Mejora de la rúbrica, mejora en la definición de los factores } \\
\text { correspondientes a las distintas materias y la evaluación de la } \\
\text { calidad de la presentación visual y verbal. }\end{array}$ & $\begin{array}{l}\text { La rúbrica es muy útil y permite evaluar con más } \\
\text { precisión. La nota media resultante de la rúbrica } \\
\text { representa el } 10 \% \text { del proyecto. }\end{array}$ \\
\hline & Mejorar el proceso de evaluación. & $\begin{array}{l}\text { Realizar una exposición pública de paneles que podrían ser } \\
\text { revisados por los colaboradores externos. }\end{array}$ & $\begin{array}{l}\text { Los colaboradores externos destacan los mejores trabajos } \\
\text { y dan una visión de la calidad del conjunto de trabajos. }\end{array}$ \\
\hline 2014 & Mejorar el proceso de evaluación. & $\begin{array}{c}\text { Propuesta de un sistema de bonificación / penalización de los } \\
\text { grupos que hay an sido capaces de cumplir con el programa o } \\
\text { calendario de trabajo previsto. }\end{array}$ & No se llego a poner en marcha. \\
\hline \multirow{2}{*}{2015} & $\begin{array}{l}\text { Introducir una nueva herramienta dentro del proceso de } \\
\text { diseño de producto que permita seguir mejorando la } \\
\text { calidad de la metodología de Proy ectos de Módulo }\end{array}$ & $\begin{array}{l}\text { Aplicación de matrices de especificaciones y factores de } \\
\text { diseño en proyectos de módulo. }\end{array}$ & $\begin{array}{c}\text { Se precisa may or implicación del profesorado, para que } \\
\text { modifiquen la herramienta según las necesidades de los } \\
\text { contenidos. }\end{array}$ \\
\hline & \begin{tabular}{|c|} 
Mejora de las habilidades de comunicación en la \\
presentación de proyectos mediante el uso de herramientas \\
audiovisuales
\end{tabular} & $\begin{array}{c}\text { Realización de la grabación en video de las presentaciones } \\
\text { intermedias y la puesta a disposición de los estudiantes del } \\
\text { material audiovisual generado }\end{array}$ & $\begin{array}{c}\text { Los alumnos pueden corregir sus defectos gracias al } \\
\text { visionado de los vídeos. No ha resultado una herramienta } \\
\text { eficaz }\end{array}$ \\
\hline \multirow{3}{*}{2016} & Mejorar el control interno del Grupo & \begin{tabular}{|c} 
Implantar un sistema de autocontrol interno mediante actas de \\
las sesiones de trabajo. En las actas se debe aportar el \% de \\
evolución del proyecto y de las distintas materias.
\end{tabular} & $\begin{array}{c}\text { Reducción de los dos picos actuales de trabajo gracias a la } \\
\text { planificación. Mayor participación activa por parte de } \\
\text { todos los integrantes. }\end{array}$ \\
\hline & Mejora del seguimiento del grupo por parte del docente. & $\begin{array}{l}\text { Seguimiento de las actas de las sesiones de trabajo por un } \\
\text { profesor tutor }\end{array}$ & $\begin{array}{l}\text { Se puede actuar antes de que los problemas internos del } \\
\text { grupo pongan en peligro sus objetivos. Se da algún caso. }\end{array}$ \\
\hline & Reconocimiento del liderazgo. & $\begin{array}{c}\text { Incentivar y premiar los comportamientos proactivos y de } \\
\text { liderazgo , así como penalizar los comportamientos } \\
\text { deshonestos. }\end{array}$ & $\begin{array}{l}\text { Las actas dan una información objetiva y contrastada } \\
\text { posteriormente que permite premiar o penalizar. No } \\
\text { todos los grupos contestan correctamente a las actas. }\end{array}$ \\
\hline \multirow{3}{*}{2017} & Mejorar las herramientas para el control interno del grupo. & $\begin{array}{l}\text { Reducción del numero de actas a cumplimentar. Cada grupo es } \\
\text { tutorizado por un profesor. }\end{array}$ & En proceso. \\
\hline & $\begin{array}{c}\text { Mejoras en las herramientas para el controlpor parte del } \\
\text { docente. }\end{array}$ & $\begin{array}{l}\text { Las actas son obligatorias antes de las presentaciones } \\
\text { intemedia y final. Control mediante la evolución del proyecto. }\end{array}$ & $\begin{array}{c}\text { En proceso. Se han realizado entrevistas con grupos de } \\
\text { alumnos que tienen o manifiestan problemas antes de } \\
\text { pasara a la fase final. }\end{array}$ \\
\hline & $\begin{array}{c}\text { Mejoras en las herramientas para el reconocimiento del } \\
\text { liderazgo. }\end{array}$ & $\begin{array}{c}\text { En las actas se debe contestar a la satisfacción respecto a la } \\
\text { evolución del proyecto, junto con la información final } \\
\text { permitirá la evaluación invididualizada. }\end{array}$ & En proceso. \\
\hline
\end{tabular}

